\title{
Supplier credits, limited liquidity, and timely demand information
}

\author{
Sabine Böckem • Ulf Schiller
}

Published online: 9 May 2010

(C) Springer-Verlag 2010

\begin{abstract}
We consider supplier-credit contracting between a manufacturer and a liquidity-constrained dealer. We show that the timeliness according to which the dealer receives demand information has a significant impact on the optimal contract. If the manufacturer cannot be sure that a dealer without liquidity has demand information when the contract is written, the optimal contract assigns the same quantity to an ignorant dealer and a dealer who knows that there are unfavorable demand conditions. However, dealers with favorable demand information are screened. If the dealer's liquidity rises, the manufacturer proposes a contract that resembles the solution of a classic adverse selection model in the spirit of Harris et al. (Manag Sci 28:604-620, 1982). For high liquidity, the optimal supplier-credit contract assigns the same quantity to an ignorant dealer and dealers who have favorable demand information whereas dealers with unfavorable demand information are screened.
\end{abstract}

Keywords Supply-chain contract · Supplier credit · Limited liquidity · Timeliness of information

\section{Introduction}

Financial management of the supply chain is not an invention of the financial crisis. However, the breakdown of the credit markets puts large members of the supply

\footnotetext{
S. Böckem $(\varangle)$

Wirtschaftswissenschaftliche Fakultät, Universität Basel,

Peter Merian-Weg 6, 4002 Basel, Switzerland

e-mail: sabine.boeckem@unibas.ch

U. Schiller

Institut für Unternehmensrechnung und Controlling, Universität Bern,

Engehaldenstrasse 4, 3012 Bern, Switzerland

e-mail: ulf.schiller@iuc.unibe.ch
} 
chain into a permanent responsibility for small and medium-sized firms. As a recent publication by Deloitte puts it: ${ }^{1}$

“[...] even after the current crisis subsides, credit is likely to remain much tighter

than in recent years, which means there will be more and more cases where sup-

ply chain financing isn't just helpful—it's essential".

This paper studies a manufacturer who must design a supply-chain contract with a liquidity-constrained dealer. The manufacturer's task is to determine the conditions of a supplier credit. Our research interest is to find conditions under which a dealer's limited liquidity has a significant influence on the shape of the optimal contract, especially on the emergence of quantity assignments that do not respond to the dealer's information about demand. We identify the timeliness of the dealer's access to information about demand as a key driver.

In our model, a liquidity-constrained dealer knows whether he has been able to timely update his knowledge about demand in the upcoming period before contracting with a manufacturer. The manufacturer faces the problem that she does not know whether she faces an informed dealer and, if informed, what the information is. Our analysis starts with benchmark cases where each of the dealer's protections at the contracting stage, limited liquidity and private demand information, is studied independently.

Limited liquidity has already been studied by Sappington (1983). Sappington shows that the optimal contract under limited liquidity ${ }^{2}$ is very similar to one where there is precontractual asymmetric information (as, e.g., in Harris et al. 1982). We supplement his results by showing that effects of limited liquidity cannot be studied convincingly in a scenario where the dealer has timely demand information at the contracting stage for sure. ${ }^{3}$ Therefore, we consider a scenario where the manufacturer is uncertain whether the dealer has timely demand information or whether the dealer is ignorant at the contracting stage.

Such potential ignorance at the contracting stage has been studied in a previous paper by Lewis and Sappington (1993); LS hereafter. LS assume that the dealer may or may not be ignorant at the contracting stage, but has no liquidity constraints. They show that the manufacturer assigns the same quantity to an informed dealer with favorable information and to an ignorant dealer.

We then consider the case where the dealer faces a liquidity constraint right from the outset. Our results are most nicely illustrated in the case where the inverse hazard rate of the demand parameter is linear. In the extreme case where the dealer has absolutely no liquidity at the outset, the optimal supply-credit contract bunches together an ignorant dealer and informed dealers who have received unfavorable information. At the

\footnotetext{
1 See Deloitte Consulting (2008): "Breathing room-Ten ideas for squeezing more working capital from your supply chain".

2 Sappington (1983) calls his assumption "limited liability". If the dealer is informed for sure, limited liability and limited liquidity lead to identical results in our model.

3 More recently, a number of papers has extended the problem to situations where the amount of the dealer's liquidity is also unknown (Che and Gale 2000; Lewis and Sappington 2000, 2001).
} 
same time, informed dealers with favorable information are screened. We then look at the optimal supply-credit contract if the dealer's liquidity gradually improves. Then, the amount of bunching is reduced. For some intermediate amount of liquidity, we obtain screening for all possible types of dealers. In this case, the form of the optimal supplier-credit contract exactly replicates the solution to the classic adverse selection model in the spirit of Harris et al. (1982). For even higher liquidity, an ignorant dealer receives the same quantity assignment as an informed dealer who knows that he has favorable information, just as in the LS model.

Our major results center around the emergence of bunching if there are liquidity constraints and/or ignorance. Therefore, we also look at the robustness of our results. We show that the curvature of the inverse hazard rate, hence the manufacturer's incentive cost, is crucial for bunching and screening patterns. For instance, the above result where there is full screening in the case of intermediate liquidity extends to the case where the inverse hazard rate is convex. However, if the inverse hazard rate is concave, full screening becomes impossible.

Our view on the problem also offers an extension to accounting research by Dye (1985), Jung and Kwon (1988) and Penno (1997). In these papers, the financial market does not know whether or not the manager possesses information. These papers make the point that the 'disclosure principle' (existence of a full screening equilibrium) might fail due to the presence of potentially ignorant managers. Instead, there will be partial pooling where good types disclose and bad types do not communicate their knowledge. The difference to our problem is that we look at contractually required reports to a creditor instead of voluntary reporting to the equity market.

The further organization of the paper is as follows. The next section introduces the model and develops the manufacturer's optimization problem. Section 3 then presents the solution in three steps. First, we look at the three benchmark settings where (i) the dealer has timely demand information for sure but no liquidity, (ii) the dealer has high liquidity but does not have timely information, and (iii) where the dealer has high liquidity but it is unknown whether or not he has timely information. Afterwards we derive the general solution. Some final discussions are in the concluding Sect. 4.

\section{The model}

There are two firms, a manufacturer (firm 1; she) and a dealer (firm 2; he). The latter sells the product on the downstream market, thus earning a revenue $R(q, \theta)=$ $\theta q+r(q)$ where $q$ denotes the number of units sold and $\theta$ is the realization of a random variable. High values of $\theta$ indicate good information. For all $\theta$, we assume that $R(0, \theta)=0$ and $R(q, \theta)$ is concave in $q$ and increasing at $q=0$. This additive form is a simple representation of revenues in an imperfect downstream market. It includes the familiar linear demand case where $R(q, \theta)=\theta q-b q^{2}, b>0$.

Prior to the contracting stage, firms share common beliefs over the distribution function $F(\theta)$ with positive density $f(\theta)>0$ over an interval $[\underline{\theta}, \bar{\theta}]$ with $\underline{\theta}>0$. 


\begin{tabular}{|c|c|c|c|}
\hline date 0 & date 1 & date 2 & date 3 \\
\hline $\begin{array}{l}\text { With prob. } p \text {, dealer } \\
\text { receives timely de- } \\
\text { mand information, } \\
s=\theta, \text { and with } \\
\text { prob. }(1-p) \text {, dealer } \\
\text { gets no information, } \\
s=s^{0}\end{array}$ & $\begin{array}{l}\text { Manufacturer } \\
\text { designs supply } \\
\text { credit contract } \\
{[q(m), t(m)]} \\
\text { Dealer accepts } \\
\text { or rejects }\end{array}$ & $\begin{array}{l}\text { Dealer reports } m \text { to } \\
\text { the manufacturer } \\
\text { Manufacturer pro- } \\
\text { duces and delivers } \\
\text { quantity } q(m)\end{array}$ & $\begin{array}{l}\text { Dealer sells } q(m) \\
\text { on the consumer } \\
\text { market } \\
\text { Afterwards dealer } \\
\text { must pay } t(m) \text { to } \\
\text { the manufacturer }\end{array}$ \\
\hline
\end{tabular}

Fig. 1 Time line

We denote the expected value of $\theta$ by $E\{\theta\}$ and assume that $F(\theta)$ satisfies a standard regularity condition. ${ }^{4}$

Assumption 1 The functions

$$
\theta \rightarrow \frac{F(\theta)}{f(\theta)} \text { and } \theta \rightarrow-\frac{1-F(\theta)}{f(\theta)}
$$

are increasing in $\theta$.

Figure 1 depicts the sequence of events. We assume that with probability $p$, the dealer receives timely demand information at date 0 . In this case, he learns the demand parameter $\theta$ before the supply-chain contract is written. With probability $(1-p)$, the dealer receives no such information before the contracting stage and learns $\theta$ only ex post at date 3 . In this case, we call the dealer ignorant at the contracting stage. Formally, we assume that the dealer obtains a signal $s$ that, with probability $p$, conveys precise information $s=\theta$. With probability $(1-p)$, the dealer receives an entirely uninformative signal $s=s^{0}$ and learns nothing. The manufacturer has no access to any information gathering technology. At any time, the value of the demand parameter $\theta$ remains unverifiable to third parties, that is, $\theta$ is even uncontractible ex post. We assume that $p$ is common knowledge.

To produce $q$, the manufacturer incurs production costs $C(q)$ with $C(0)=C^{\prime}(0)=$ $0, C^{\prime}(q>0)>0$, and $C^{\prime \prime}(q) \geq 0$.

At the outset, the dealer has limited funds that determine his liquidity. Denote these initial liquid funds by $K$. If $K$ is low, the dealer may not be able to pay the manufacturer at the time of delivery. Then, the manufacturer must act as a creditor. The supplier credit can only be refunded after $q$ has been sold on the downstream market. To avoid further complexity, we assume that the dealer always fully sells the available quantity and that no retention of title clause is feasible, for instance, because the country's jurisdiction does not support such clauses. ${ }^{5}$

\footnotetext{
${ }^{4}$ Usually, only the second of the above properties is assumed. This is because in a standard hidden-type agency model, incentive constraints are binding towards bad types ("downward binding"). In our setting, also "upward" binding incentive constraints may occur. Thus, we need both regularity conditions.

5 Retention of title clauses are particularly prevalent in the Germanic countries. In other European countries and in North America they appear to be less common. There may be a variety of reasons for this, most importantly seems to be the separation of contract right and property right (Abstraktionsprinzip) in the
} 
The supply-chain contract specifies some amount $t$ that must be refunded at the end of the period. The manufacturer's and the dealer's profits, $\Pi_{1}$ and $\Pi_{2}$, then read as

$$
\Pi_{1}=t-C(q) \text { and } \Pi_{2}=\theta q+r(q)-t .
$$

At date 1, the manufacturer suggests a supply credit contract to the dealer. We denote a supply credit contract by $[q(\cdot), t(\cdot)]$ where $q(\cdot)$ is a quantity assignment and $t(\cdot)$ is the dealer's payment obligation. Without loss of generality, we can invoke the revelation principle and concentrate on direct truth-telling mechanisms (Myerson 1982). That is, the manufacturer asks the dealer to report the information $s$ at date 2 . For each possible report $m \in\left\{s^{0}\right\} \cup[\underline{\theta}, \bar{\theta}]$, the manufacturer designs a specific quantity assignment $q(m)$ and an according payment obligation $t(m)$. In a revelation contract, the dealer reports truthfully, i.e., $m=s$ for all $s \in\left\{s^{0}\right\} \cup[\underline{\theta}, \bar{\theta}]$. Put otherwise, the dealer with information $s$ must prefer a supply credit contract $[q(s), t(s)]$ to any other contract $\left[q\left(s^{\prime}\right), t\left(s^{\prime}\right)\right]$ in the menu of contracts that has been specified by the manufacturer at date 1 . To shorten notation, we define the contract that is designed for an ignorant dealer as $\left[q^{0}, t^{0}\right] \equiv\left[q\left(s^{0}\right), t\left(s^{0}\right)\right]$.

The manufacturer faces two difficulties at date 1 . First, the menu of supply-credit contract proposals must reflect the fact that the dealer is possibly ignorant about $\theta$. Second, the payment obligation $t$ must never exceed the dealer's total verifiable liquidity at date 3 when $q$ has been sold on the downstream market.

The second of the manufacturer's difficulties deserves a deeper discussion. As emphasized, e.g. by Cachon (2003) and Cachon and Lariviere (2001), the set of feasible payment obligations $t(m)$ crucially depends on the compliance regime. Sanctions must prevent that after having signed some contract $(q(m), t(m))$, the dealer declares himself unable to pay $t(m)$. More concretely, contract enforcement depends on the interplay of civil law and criminal law. With unlimited verifiable liquidity, ex-post moral hazard can easily be avoided by specifying prohibitive contractual sanctions; hence the rules of criminal law are irrelevant for ensuring that the dealer fulfills his payment obligation. However, with limited liquidity, contractual sanctions are limited to the amount of verifiably available liquid funds. These funds are given by the minimum possible revenue, $R(q(m), \underline{\theta})=\underline{\theta} q(m)+r(q(m))$, plus the dealer's initial liquid funds $K$. We assume that the dealer can secretly consume any revenue that is earned in excess. It follows that limited liquidity seriously affects the set of feasible contracts. If it cannot be verified that the dealer's liquid funds at date 3 exceed $R(q(m), \underline{\theta})+K$, any initial payment obligation $t\left(m=s^{0}\right)$ that exceeds $R(q(m), \underline{\theta})+K$ cannot be enforced.

Enforceability of contracts is also supported by criminal law, though. Suppose for instance that, at date 2 , a dealer declares to know that market conditions are extremely good, i.e., $m=\bar{\theta}$. At the time of payment (date 3), he declares himself unable to pay. The dealer may, for instance, pretend that his initial announcement was false and the true realization was $\underline{\theta}$. In such a case, he admits fraud $(m \neq s)$ and sanctions according

Footnote 5 continued

Germanic countries. In most other countries, retention of title clauses expire when the retailer sells the good to a third party or are not even feasible in the first place. 
to criminal law will be imposed. We assume that punishments are prohibitively harsh such that it is never rational for the dealer to declare $m \neq s$ ex post. ${ }^{6}$

Assumption 2 Criminal law classifies the dealer's reporting behavior as fraud if and only if the dealer must admit that $m \neq s$. Fraud is punished such that the dealer's perceived disadvantage from the sanction is prohibitively high.

Under Assumption 2, a report of $m=s^{0}$ will never be classified as fraud because the dealer has admitted that he is ignorant. Given that there is common knowledge about $K$ and the probability $p$, the manufacturer designs the supply-credit contract. She aims at maximizing her own expected profit,

$$
\begin{aligned}
& \max _{\substack{q(s), t(s) \\
s \in\left\{s^{0}\right\} \cup[\underline{\theta}, \bar{\theta}]}} E\left\{\Pi_{1}\right\}=p \int_{\underline{\theta}}^{\bar{\theta}}[t(\theta)-C(q(\theta))] f(\theta) \mathrm{d} \theta \\
& +(1-p)\left[t^{0}-C\left(q^{0}\right)\right],
\end{aligned}
$$

subject to a set of constraints that we introduce below.

Given an actual demand parameter $\theta$, define the dealer's profit after having reported $m$ by $\Pi_{2}(m \mid \theta)$. There are two kinds of participation constraints. An informed dealer will accept the contract only if he receives a non-negative profit under truth telling,

$$
\Pi_{2}(\theta \mid \theta)=\theta q(\theta)+r(q(\theta))-t(\theta) \geq 0 \quad \forall \theta \in[\underline{\theta}, \bar{\theta}] .
$$

Similarly, an ignorant dealer will participate if he expects non-negative profits,

$$
E_{\theta}\left\{\Pi_{2}\left(s^{0} \mid \theta\right)\right\}=E\{\theta\} q^{0}+r\left(q^{0}\right)-t^{0} \geq 0
$$

Since the informed dealer is protected by constraint (3), limited liquidity has bite if the manufacturer faces the ignorant dealer. The latter's ex-post profits may turn out to be negative. Therefore, it must hold

$$
\Pi_{2}\left(s^{0} \mid \theta\right)=\theta q^{0}+r\left(q^{0}\right)-t^{0} \geq-K \quad \forall \theta \in[\underline{\theta}, \bar{\theta}]
$$

which is implied by

$$
\Pi_{2}\left(s^{0} \underline{\theta}\right)=\underline{\theta} q^{0}+r\left(q^{0}\right)-t^{0} \geq-K .
$$

\footnotetext{
6 Notice that if the dealer has limited liquid funds, these sanctions must be of non-monetary nature (e.g. imprisonment). The manufacturer cannot replicate such punishments through a contract. The basic message of our model carries over to a model with ex post uncertainty with $R(q, \theta)=\theta q+r(q)+\epsilon$ if the variance of the ex post noise $\epsilon$ is sufficiently small, such that, in the presence of extreme deviations of $E\{R(q(m), m)\}$ from (verifiable) ex post revenue, a court will judge that fraud has taken place "beyond a reasonable doubt".
} 
Notice that with Assumption 2, it is not optimal to specify any payment obligation higher than $t^{0}=\underline{\theta} q^{0}+r\left(q^{0}\right)+K$ because the dealer will always be able to claim that the true state of nature was $\underline{\theta}$.

Now consider the incentive constraints. First, the informed dealer should report truthfully, that is,

$$
\Pi_{2}(\theta \mid \theta) \geq \Pi_{2}(m \mid \theta) \quad \forall(\theta, m) \in[\underline{\theta}, \bar{\theta}]^{2} .
$$

Moreover, the informed dealer should not claim to be ignorant,

$$
\Pi_{2}(\theta \mid \theta) \geq \Pi_{2}\left(s^{0} \mid \theta\right) \quad \forall \theta \in[\underline{\theta}, \bar{\theta}] .
$$

Finally, an ignorant dealer should not claim to be informed,

$$
E_{\theta}\left\{\Pi_{2}\left(s^{0} \mid \theta\right)\right\} \geq E_{\theta}\left\{\Pi_{2}(m \mid \theta)\right\} \quad \forall m \in[\underline{\theta}, \bar{\theta}] .
$$

In the following we will make extensive use of the result that the informed dealer's incentive constraint (6) is satisfied if and only if the quantity assignment $q(\theta)$ is nondecreasing in $\theta$. We call this the monotonicity constraint (see Guesnerie and Laffont 1984, or Theorem 7.3 in Fudenberg and Tirole 1991).

\section{Optimal supply credit contracts}

\subsection{Three benchmark cases}

Before we come to the solution to the manufacturer's general problem we sketch the solutions of three benchmark cases. In the first, the probability $p$ of facing an informed dealer equals one. In the second, we assume that the dealer is ignorant for sure but has unlimited liquidity. In the third, liquidity is again unlimited, but the probability $p$ of having an informed dealer is strictly between zero and one. In all these cases, contract enforcement and compliance play no active role.

First, consider the case of $p=1$. In this case the constraints (4), (7), and (8) are irrelevant and we are back to the standard situation of the literature (e.g. Harris et al. 1982). The solution to the problem is well known. Denote the optimal quantity assignment rule by $q^{p=1}(\theta)$.

Lemma 1 Suppose that $p=1$. For all $K \geq 0$ the optimal quantity assignment rule $q^{p=1}(\theta)$ maximizes

$$
\int_{\underline{\theta}}^{\bar{\theta}}\left[\theta q(\theta)+r(q(\theta))-C(q(\theta))-q(\theta) \frac{1-F(\theta)}{f(\theta)}\right] f(\theta) \mathrm{d} \theta
$$


and is given by

$$
\theta+r^{\prime}\left(q^{p=1}(\theta)\right)-C^{\prime}\left(q^{p=1}(\theta)\right)-\frac{1-F(\theta)}{f(\theta)}=0 \quad \forall \theta \in[\underline{\theta}, \bar{\theta}] .
$$

Proof See Appendix.

The manufacturer's objective function (9) equals the expected total supply chain profit, $E\{R(q(\theta), \theta)-C(q(\theta))\}$ minus expected incentive costs, $E\{q(\theta)(1-F(\theta)) /$ $f(\theta)\}$. These incentive costs arise because the dealer can protect himself against full extraction of earned revenue by claiming that demand is low. Hence, a dealer in a favorable market will only reveal the true realization of $\theta$ if he is compensated for the foregone benefit of cheating.

The quantity assignment rule $q^{p=1}(\theta)$ is chosen such that, for every $\theta$, marginal revenue $\theta+r^{\prime}(q(\theta))$ equals marginal virtual cost, $C^{\prime}(q(\theta))+(1-F(\theta)) / f(\theta)$. In what follows, the global shape of the marginal incentive cost $(1-F(\theta)) / f(\theta)$ will play a crucial role for the characterization of our main results.

As a further characteristic of $q^{p=1}(\theta)$, no efficiency distortion arises if the demand parameter takes it most favorable realization, i.e., $q^{p=1}(\bar{\theta})=q^{\mathrm{FB}}(\bar{\theta})$ where $q^{\mathrm{FB}}(\theta)$ denotes the First-Best quantity assignment, i.e., it solves $\theta+r^{\prime}\left(q^{\mathrm{FB}}(\theta)\right)=C^{\prime}\left(q^{\mathrm{FB}}(\theta)\right)$. Last, limited liquidity plays no active role in the standard scenario, that is, a change in $K$ does not affect the results. Under Assumption 2, the dealer will fulfill his payment obligations $t(\theta)$ in all states of nature even if he has had no liquid funds at the outset, i.e., $K=0$, because otherwise his behavior is classified as fraud.

Now consider the second benchmark case where $K$ is "very large" and $p=0$. Since the manufacturer knows for sure that the dealer is ignorant about $\theta$, she assigns the efficient quantity for the expected type, i.e., $q^{p=0}\left(s^{0}\right)=q^{\mathrm{FB}}(E\{\theta\})$.

We next turn to our third benchmark case where $0<p<1$, but, again, $K$ is "very large" such that constraint (5) is not binding. The solution to this special case of the problem has been derived by Lewis and Sappington (1993, LS) before. LS show that an ignorant dealer will get the same quantity assignment as an informed dealer who knows that $\theta=E\{\theta\}$. This result extends to our problem and we will use it without further proof. Technically spoken, the possibility that the dealer is ignorant introduces a mass point into the distribution of $\theta$ and the solution has to trade off effects of this mass point against effects occurring to the continuum of informed dealers. We denote the resulting quantity assignment pattern as $q^{\mathrm{LS}}(\theta)$.

As LS do not incorporate a liquidity constraint, they implicitly assume that $K$ is always sufficient, or that it exceeds a lower bound $K_{\mathrm{LS}}$. $K_{\mathrm{LS}}$ is given by

$$
\begin{aligned}
K_{\mathrm{LS}} & =-\Pi_{2}\left(s^{0} \mid \underline{\theta}\right)=-\underline{\theta} \cdot q^{\mathrm{LS}}(E\{\theta\})-r\left(q^{\mathrm{LS}}(E\{\theta\})\right)+t^{\mathrm{LS}}(E\{\theta\}) \\
& =[E\{\theta\}-\underline{\theta}] q^{\mathrm{LS}}(E\{\theta\})-\int_{\underline{\theta}}^{E\{\theta\}} q^{\mathrm{LS}}(\vartheta) \mathrm{d} \vartheta .
\end{aligned}
$$

If $K>K_{\mathrm{LS}}$, the limited-liquidity constraint (5) is slack. 


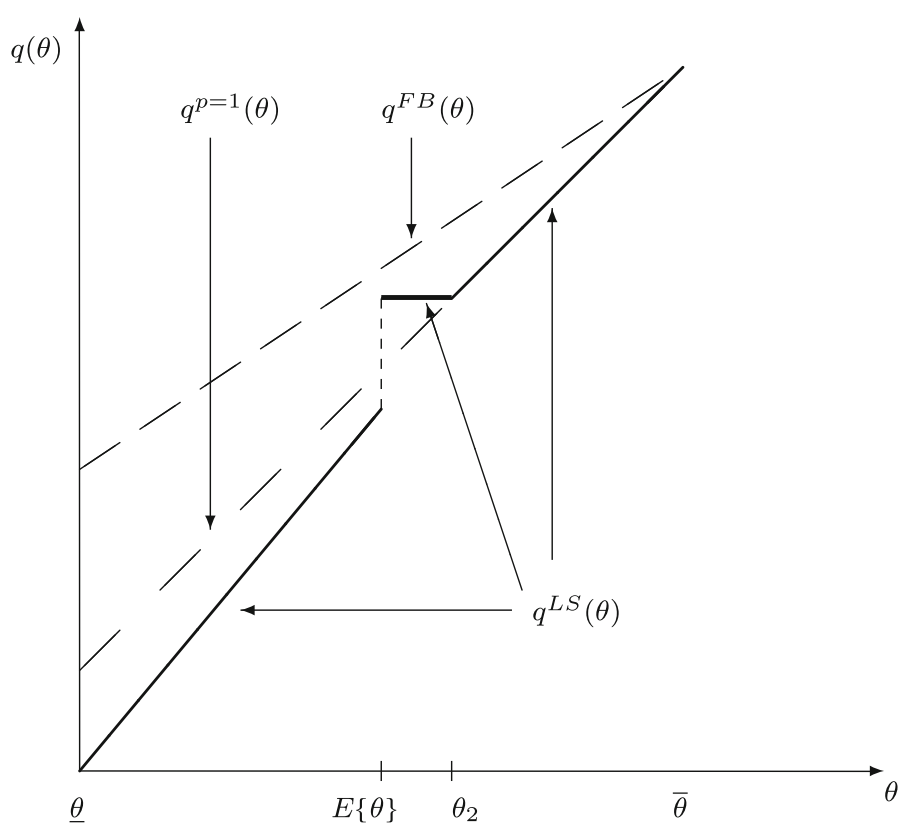

Fig. 2 The First-Best (FB), the $p=1$, and the Lewis-Sappington (LS) benchmark solutions

The following Lemma 2 formally states and Fig. 2 above illustrates the solution that rests on the following reasoning. We start from a naïve combination of the above benchmark results for $p=1$ and $p=0$, but now assume that $p \in(0,1)$. Obviously, such a naïve combination, $q(E\{\theta\})=q^{\mathrm{FB}}(E\{\theta\})$ and $q\left(\theta \neq E(\{\theta\})=q^{p=1}(\theta)\right.$, would lead to false reporting. To prevent a dealer with some information $\theta^{\prime}>E\{\theta\}$ from claiming to be ignorant, the manufacturer must make sure that $q\left(\theta^{\prime}\right) \geq q(E\{\theta\})$. Since there exists a nonempty interval $\Theta^{\prime}$ with $q^{p=1}\left(\theta \in \Theta^{\prime}\right)<q^{\mathrm{FB}}(E\{\theta\})$, the manufacturer must raise $q\left(\theta \in \Theta^{\prime}\right)$ above the benchmark level for the $p=1$ case. Simultaneously, the resulting inefficiency will be mitigated if the manufacturer reduces $q(E\{\theta\})$ below its First-Best level, thus also reducing the length of the interval with $q^{p=1}\left(\theta^{\prime}\right)<q(E\{\theta\})$. Hence, there is bunching over an interval $\left[E\{\theta\}, \theta_{2}\right]$. Moreover, the manufacturer will care about misreporting incentives for an ignorant dealer who might be tempted to claim that $\theta<E\{\theta\}$. The associated incentive cost is higher than in the $p=1$ benchmark case: it consists of the incentive cost for an informed dealer plus an additional ignorance cost for an uninformed dealer. These incentive costs, in sum, lead to a higher distortion for an informed dealer with $\theta<E\{\theta\}$. Therefore, $q^{\mathrm{LS}}(\theta<E\{\theta\})$ is depressed below the level of the benchmark solution $q^{p=1}(\theta)$.

Lemma 2 (Lewis and Sappington 1993, Proposition 1) Suppose that $p \in(0,1)$. Then, there exist a critical level of liquid funds, $K_{\mathrm{LS}}$, and a boundary level of the demand parameter, $\theta_{2}>E\{\theta\}$, such that for all $K \geq K_{\mathrm{LS}}$, the optimal quantity assignment $q^{\mathrm{LS}}(\theta)$ is given by

1. $q^{\mathrm{LS}}(\theta)=q^{p=1}(\theta) \quad \forall \theta \in\left[\theta_{2}, \bar{\theta}\right]$,

2. $q^{\mathrm{LS}}(\theta)=q^{\mathrm{LS}}(E\{\theta\}) \in\left(q^{p=1}(E\{\theta\}), q^{\mathrm{FB}}(\theta)\right) \quad \forall \theta \in\left[E\{\theta\}, \theta_{2}\right]$ 
3. $q^{\mathrm{LS}}(\theta)<q^{p=1}(\theta) \quad \forall \theta \in[\underline{\theta}, E\{\theta\})$

where, for this interval, $q^{\mathrm{LS}}(\theta)$ solves

$$
\theta+r^{\prime}(q(\theta))-C^{\prime}(q(\theta))-\frac{1-F(\theta)}{f(\theta)}-\frac{1-p}{p f(\theta)}=0 .
$$

Proof See Lewis and Sappington (1993).

\subsection{Solution to the general problem}

We now turn to the general case where the dealer's liquidity $K$ does not exceed $K_{\mathrm{LS}}$ and where $0<p<1$. Using a result by LS, the manufacturer's problem (2)-(8) is considerably simplified because of the following Lemma.

Lemma 3 The manufacturer assigns the same quantity to an ignorant dealer and an informed dealer who knows that the realization of $\theta$ happens to be exactly $E\{\theta\}$,

$$
q^{0}=q(E\{\theta\}), \quad t^{0}=t(E\{\theta\}) .
$$

Proof The proof is identical to the one in LS.

Lemma 3 tells that an ignorant dealer is treated identically to a dealer who knows that his demand parameter is equal to $\theta=E\{\theta\}$. The implication of this result is that the manufacturer can treat the problem as one where the probability distribution of the demand parameter has a mass point at $\theta=E\{\theta\}$.

This result is helpful in reducing the number of constraints. First, constraint (4) is satisfied along with condition (3), and constraints (7) and (8) become special cases of condition (6). In addition, using an element in the proof of Lemma 1, the participation constraint (3) is implied by the simpler condition $\Pi_{2}(\underline{\theta} \mid \underline{\theta}) \geq 0$ and the incentive compatibility constraint (6) may be replaced by the monotonicity constraint. These observations enable us to restate the manufacturers maximization problem as follows.

$$
\max _{\left\{\begin{array}{c}
q(s), t(s) \\
s \in\left\{s^{0}\right\} \cup[\underline{\theta}, \bar{\theta}]
\end{array}\right\}} E\left\{\Pi_{1}(\theta)\right\}=p \int_{\underline{\theta}}^{\bar{\theta}}[t(\theta)-C(q(\theta))] f(\theta) d \theta+(1-p)\left[t^{0}-C\left(q^{0}\right)\right]
$$

$$
\begin{array}{ll}
\text { subject to } & \Pi_{2}(\underline{\theta} \mid \underline{\theta}) \geq 0, \\
& \underline{\theta} q^{0}+r\left(q^{0}\right)-t^{0} \geq-K, \\
& q(\theta) \text { non-decreasing in } \theta .
\end{array}
$$

In what follows we denote the Lagrangian multiplier to constraint (14) by $\lambda$ and to constraint (15) by $\mu, \lambda, \mu \geq 0$. 
Proposition 1 If $0 \leq K \leq K_{\mathrm{LS}}$, both constraints, (14) and (15), are binding with $\lambda=1-\mu$. Moreover, $\lambda=0$ if and only if $K=0$ and $\mu=0$ if and only if $K=K_{\mathrm{LS}}$.

The result in Proposition 1 follows from the fact that a rise in $K$ reduces $\mu$ because, on the one hand, the limited liquidity constraint is relaxed. On the other hand, relaxing the limited liquidity constraint in the manufacturer's optimization problem increases the relevance of the participation constraint that was only weakly binding in the extreme case of $K=0$. Thus, $\lambda$ is increasing in $K$.

To save notation, let $\mathbf{1}_{\Sigma}$ be 1 if the statement $\Sigma$ is true and 0 otherwise. The steps along the proof also enable us to rewrite the manufacturer's problem as to maximize ${ }^{7}$

$$
\begin{aligned}
& p \int_{\underline{\theta}}^{\bar{\theta}}\left[\theta q(\theta)+r(q(\theta))-C(q(\theta))-q(\theta)\left(\frac{1-F(\theta)}{f(\theta)}-\frac{(p-\lambda) \mathbf{1}_{\theta \leq E\{\theta\}}}{p f(\theta)}\right)\right] f(\theta) \mathrm{d} \theta \\
& \quad+(1-p)\left[\underline{\theta} q^{0}+r\left(q^{0}\right)-C\left(q^{0}\right)\right]-(p-\lambda)[E\{\theta\}-\underline{\theta}] q^{0}+(1-\lambda) K,
\end{aligned}
$$

with respect to $q(\theta)$ and $q^{0}$, subject to $q^{0}=q(E\{\theta\})$ and $q(\theta)$ non-decreasing in $\theta$.

The new objective function reflects the different effects that enter the manufacturer's optimization problem. First, the new objective function is a weighted average of the benefits from contracting with the dealer in the state where demand information has arrived timely and where the dealer is ignorant. Second, opportunity costs that arise from the dealer's limited liquidity enter the problem. Third, similar to the LS problem, contracting with a dealer who knows that demand is low induces additional

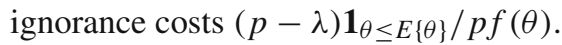

For a first step towards the solution, we temporarily ignore the monotonicity requirement. Then, pointwise maximization delivers a set of preliminary 'first-order conditions'.

$$
\begin{array}{ll}
\text { If } \theta>E\{\theta\} & \theta+r^{\prime}\left(q^{u}(\theta)\right)-C^{\prime}\left(q^{u}(\theta)\right)-\frac{1-F(\theta)}{f(\theta)}=0 \\
\text { if } \theta<E\{\theta\} & \theta+r^{\prime}\left(q^{u}(\theta)\right)-C^{\prime}\left(q^{u}(\theta)\right)-\left(\frac{1-F(\theta)}{f(\theta)}-\frac{p-\lambda}{p} \frac{1}{f(\theta)}\right)=0 \\
\text { if } \theta=E\{\theta\} & (1-p)\left[\underline{\theta}+r^{\prime}\left(q^{u}(E\{\theta\})\right)-C^{\prime}\left(q^{u}(E\{\theta\})\right)\right]-(p-\lambda)[E\{\theta\}-\underline{\theta}]=0
\end{array}
$$

Here, $q^{u}(\theta)$ denotes the solution to these three equations where the monotonicity requirement is ignored. To shorten notation, define $\underline{q} \equiv \lim _{\epsilon \rightarrow 0}\left[q^{u}(\theta-\epsilon)\right]$ and $\bar{q} \equiv \lim _{\epsilon \rightarrow 0}\left[q^{u}(\theta+\epsilon)\right]$ (where $\epsilon>0$ ). The solution to the relaxed maximization program violates the monotonicity requirement in many cases.

In order to illustrate the impact of limited liquidity and the violation of the monotonicity constraint as clearly as possible, consider the case of $K=0$ and hence $\lambda=0$.

\footnotetext{
7 Compare Lemma 5 in the Appendix.
} 


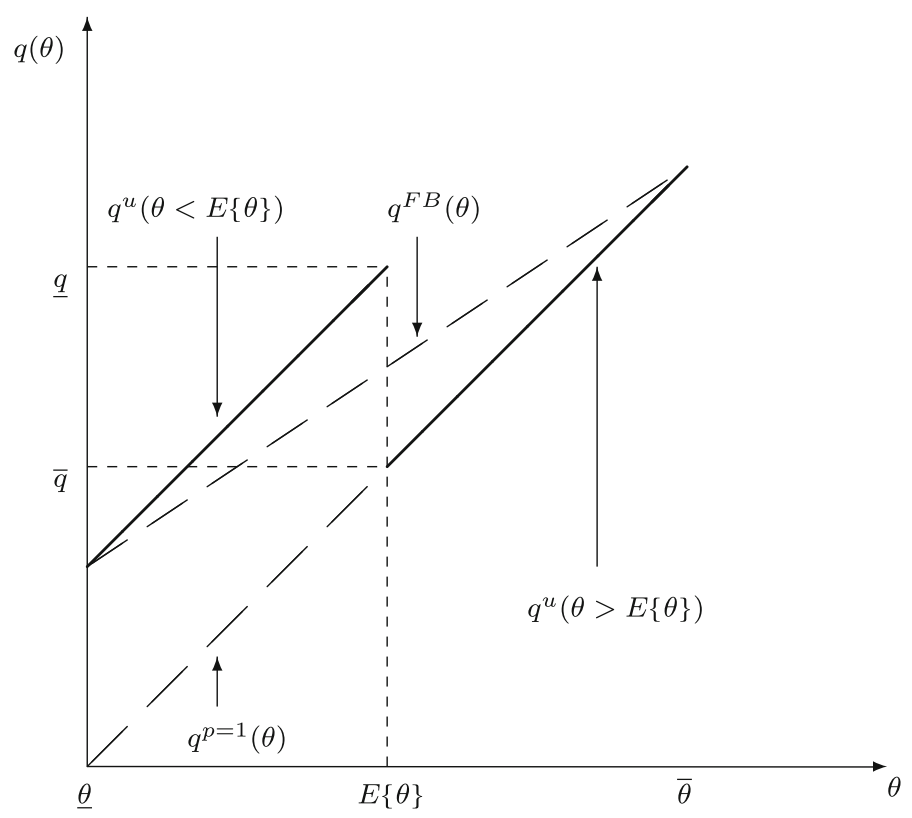

Fig. 3 Countervailing incentives in the case of $K=0$

Condition (17) reveals that the upward sided limit gives the usual $p=1$ benchmarkcase rule

$$
\lim _{\epsilon \rightarrow 0}\left[E\{\theta\}+\epsilon+r^{\prime}\left(q^{u}(E\{\theta\}+\epsilon)\right)-C^{\prime}\left(q^{u}(E\{\theta\}+\epsilon)\right)\right]=\frac{1-F(E\{\theta\})}{f(E\{\theta\})}>0
$$

whereas, from condition (18), the left-hand sided limit is

$$
\lim _{\epsilon \rightarrow 0}\left[E\{\theta\}-\epsilon+r^{\prime}\left(q^{u}(E\{\theta\}-\epsilon)\right)-C^{\prime}\left(q^{u}(E\{\theta\}-\epsilon)\right)\right]=-\frac{F(E\{\theta\})}{f(E\{\theta\})}<0 .
$$

It follows that $q>\bar{q}$. The situation is graphed in Fig. 3. The manufacturer has an incentive to assign a quantity below the First-Best level to an informed dealer with favorable information and to assign a quantity above the First-Best level to an informed dealer with unfavorable information.

To gather the intuition for this, note first that in the absence of a potentially ignorant dealer, the incentive constraints are (locally) binding downwards, i.e., a dealer with favorable information must be compensated for not pretending to have unfavorable information. Now, take the possibility of an ignorant dealer into account. Because of the protection by limited liquidity, the latter will earn an expected profit equal to $[E\{\theta\}-\underline{\theta}] q^{0}$. Imagine that, in this situation, the manufacturer proposes a contract that resembles the solution to the $p=1$ benchmark case. Then, a dealer with 
information $s=E\{\theta\}-\varepsilon(\varepsilon>0$ "small") could gather an enormous increase in profit by slightly overstating the demand parameter. Therefore, contrary to the $p=1$ benchmark case, incentive constraints are upward binding for some $\theta<E\{\theta\}$. It follows that the manufacturer will want to increase the quantity $q(\theta)$ to a higher level than $q^{\mathrm{FB}}(\theta)$ rather than decrease it. Naturally, this effect is absent for a dealer who has more favorable information than $E\{\theta\}$. For the latter types of the dealer, the manufacturer prefers the usual under-trade assignment. Hence, for $K=0$, the quantity assignment for the relaxed problem jumps downward at $E\{\theta\}$. This is known as countervailing-incentive effect (see Lewis and Sappington 1989; Jullien 2000).

Returning to the general case, $\lambda \geq 0$ or $K \geq 0$, the preliminary quantity assignment may either jump upward or downward at $E\{\theta\}$, depending on the relative size of $\lambda$ and $p$. The above sketch shows that for a given $p$, there is a downward jump $(q>\bar{q})$ if $K$ is small. On the other hand, the LS benchmark result in Lemma 2 tells us that there is an upward jump $(q<\bar{q})$ if $K$ is sufficiently large.

The presence of a potentially ignorant dealer raises a second monotonicity problem of the quantity assignment, though. While the above countervailing-incentive effect just affects the informed types of dealers around $E\{\theta\}$, it remains to clarify how the manufacturer wishes to set the quantity assignment for an ignorant dealer. It is obvious that the manufacturer's benefit from dealing with an ignorant dealer is different from the benefit of dealing with an informed dealer. On the one hand, distorting the quantity scheme at $E\{\theta\}$ is costly to the manufacturer because the total surplus is diminished. On the other hand, by raising the quantity towards the First-Best level, the manufacturer must leave higher expected rents to informed types of the dealer with $\theta>E\{\theta\}$.

If the monotonicity constraint is violated, we need to "smooth" the quantity assignment scheme and bunching will occur for some subinterval $\left[\theta_{1}, \theta_{2}\right] \subset[\underline{\theta}, \bar{\theta}]$. Departing from the preliminary "solution", $q^{u}(\theta)$, we also have to optimize over the bunching region. As the potential jump of the preliminary solution $q^{u}(\theta)$ occurs at $E\{\theta\}$, this point will clearly be an element of the bunching region. To solve for the optimal bunching region and, hence, $q^{0}$, define the maximum value function

$$
\begin{aligned}
W^{*}\left(q^{0}\right)= & E\left\{\Pi_{1}+\Pi_{2}-q^{u}(\theta) \frac{1-F(\theta)-p+\lambda}{f(\theta)} \mid \theta<\theta_{1}\right\} \\
& +E\left\{\Pi_{1}+\Pi_{2}-q^{0} \frac{1-F(\theta)-p+\lambda}{f(\theta)} \mid \theta_{1} \leq \theta<E\{\theta\}\right\} \\
& +E\left\{\Pi_{1}+\Pi_{2}-q^{0} \frac{1-F(\theta)}{f(\theta)} \mid E\{\theta\} \leq \theta<\theta_{2}\right\} \\
+ & E\left\{\Pi_{1}+\Pi_{2}-q^{u}(\theta) \frac{1-F(\theta)}{f(\theta)} \mid \theta \geq \theta_{2}\right\} .
\end{aligned}
$$

The optimal bunching quantity assignment $q^{0}$ is given as the solution to

$$
\begin{aligned}
& \max _{q^{0}} p \cdot W^{*}\left(q^{0}\right)+(1-p) \cdot\left[\underline{\theta} q^{0}+r\left(q^{0}\right)-C\left(q^{0}\right)\right] \\
& -(p-\lambda) \cdot[E\{\theta\}-\underline{\theta}] \cdot q^{0}+(1-\lambda) \cdot K
\end{aligned}
$$


The corresponding first-order condition reads ${ }^{8}$

$$
\begin{aligned}
& (1-p) \underline{\theta}+\left[1-p+p\left(F\left(\theta_{2}\right)-F\left(\theta_{1}\right)\right)\right]\left[r^{\prime}\left(q^{0}\right)-C^{\prime}\left(q^{0}\right)\right] \\
& \quad-p \cdot\left[\theta_{2}\left(1-F\left(\theta_{2}\right)\right)-\theta_{1}\left(1-F\left(\theta_{1}\right)\right)\right]-(p-\lambda)\left(\theta_{1}-\underline{\theta}\right)=0 .
\end{aligned}
$$

Now consider how the solution to (22), $q^{0}$, reacts to an increase of liquidity $K$. In this case, the ignorant dealer is less protected and will earn lower profits. The consequences are twofold. First, the quantity that the manufacturer wishes to assign to the ignorant dealer will be moved towards a more efficient level. Technically, the preliminary quantity $q^{u}(E\{\theta\})$ rises. Second, a rise in $K$ directly affects the behavior of an informed dealer who knows that $\theta<E\{\theta\}$. As the rent for the ignorant type is reduced, there are less incentives to overstate the own type. This enables the manufacturer to reduce the preliminary over-trade assignment. As a result, the preliminary quantity assignment $q^{u}(\theta<E\{\theta\})$ is reduced. This again reduces the length of the bunching area below $\theta=E\{\theta\}$.

Finally, note that for $K \rightarrow K_{\mathrm{LS}}$, the protection of the ignorant dealer by the limited-liability constraint vanishes, $\mu=0$ (and $\lambda=1$ ). If, in this case, there was no interaction with the informed types, the manufacturer would be willing to set $q^{0}$ equal to the First-Best level. However, because there is interaction, the manufacturer would have to leave high information rents to an informed dealer with $\theta>E\{\theta\}$ if $q^{0}$ was set equal to $q^{\mathrm{FB}}(E\{\theta\})$. Hence, $q^{0}$ is set below the efficient level and the quantity assignments to types below $E\{\theta\}$ are set even below $q^{p=1}(\theta)$.

Given the above effects, the final question is about the relative speeds with which the downward and upward movements of $q$ and $q^{u}(E\{\theta\})$ occur, once $K$ rises. Generally, it is very unlikely that $q$ and $q^{u}(E\{\theta \overline{\}})$ cross each other at some value such that, by coincidence, they simultaneously match the upper limit quantity $\bar{q}$. In this very special case, no bunching occurs and the quantity assignment schedule $q^{u}(\theta)$ from the relaxed problem is part of the optimal solution.

To prepare the analysis of the resulting movements of $\underline{q}$ and $q^{u}(E\{\theta\})$ when $K$ rises, we introduce the following definition.

Definition 1 The inverse hazard rate $(1-F(\cdot)) / f(\cdot)$ is called

$$
\begin{aligned}
& \text { Jensen convex if and only if } E\left\{\frac{1-F(\theta)}{f(\theta)}\right\} \geq \frac{1-F(E\{\theta\})}{f(E\{\theta\})}, \\
& \text { Jensen concave if and only if } E\left\{\frac{1-F(\theta)}{f(\theta)}\right\} \leq \frac{1-F(E\{\theta\})}{f(E\{\theta\})},
\end{aligned}
$$

and Jensen linear if and only if it is Jensen concave and Jensen convex. As usual, we speak of strict Jensen convexity/concavity if and only if strict inequalities apply.

\footnotetext{
8 See the proof to Lemma 6 in the Appendix.
} 
Remark Note that the case of Jensen convexity covers all symmetric and single-peaked density functions. ${ }^{9}$ Moreover, a uniform distribution has a Jensen linear inverse hazard rate.

In order to describe the quantity assignment, we also use the following definition.

Definition 2 If the optimal bunching quantity $q^{0}$ is such that $\theta_{1}<E\{\theta\}$ and $\theta_{2}=$ $E\{\theta\}$, we call the bunching regime downward sided.

If $q^{0}$ induces $\theta_{1}<E\{\theta\}$ and $\theta_{2}>E\{\theta\}$, we call the bunching regime double sided. sided.

If $q^{0}$ is such that $\theta_{1}=E\{\theta\}$ and $\theta_{2}>E\{\theta\}$, we call the bunching regime upward

Given these definitions, consider the case of Jensen linearity closer. For this special case, there exists some intermediate $K$ such that the same quantity assignment emerges as in the $p=1$ benchmark case. We state this as our first central result.

Theorem 1 Let the inverse hazard rate be Jensen linear. For any given value of $p$, there exists $\hat{K}$ such that $\mu(\hat{K})=(1-p)$ and $\lambda(\hat{K})=p$. We obtain downward sided bunching for all $K<\hat{K}$ while there is upward sided bunching for all $K>\hat{K}$. For $K=\hat{K}$, the optimal quantity assignment equals $q^{p=1}(\theta)$.

The nice properties of the case with Jensen linearity can be easily seen from inspection of the 'preliminary first-order conditions' (17), (18), and (19). If $\lambda(K)=p$, it is straightforward to see that $\underline{q}=\bar{q}$. To see that condition (19) completes the continuous quantity assignment rule, rewrite the equation as

$$
\underline{\theta}+r^{\prime}\left(q^{0}\right)-C^{\prime}\left(q^{0}\right)=E\{\theta\}+r^{\prime}\left(q^{0}\right)-C^{\prime}\left(q^{0}\right)-E\left\{\frac{1-F(\theta)}{f(\theta)}\right\}=0
$$

which, under Jensen linearity, is equivalent to

$$
E\{\theta\}+r^{\prime}\left(q^{0}\right)-C^{\prime}\left(q^{0}\right)-\frac{1-F(E\{\theta\})}{f(E\{\theta\})}=0 .
$$

Suppose that $p$ drops below $\lambda(K)$. Now, the manufacturer must decide where to put the bunching region. If the probability that the manufacturer faces an informed dealer is low, the necessary rent that induces truth-telling by an informed dealer with $\theta>E\{\theta\}$ becomes relatively large. To limit this rent, the quantity assignment for an informed dealer with $\theta<E\{\theta\}$ is depressed even below the $p=1$ assignment and there is bunching for types above $E\{\theta\}$. On the other hand, suppose that $p>\lambda(K)$. Then, the probability of facing an informed dealer is large, but limited liquidity is a relatively serious issue. As a consequence, the manufacturer has an incentive to reduce

\footnotetext{
9 If $f(\cdot)$ is symmetric we have $f(E\{\theta\}-a)=f(E\{\theta\}+a)$ which implies $1-F(E\{\theta\})=\frac{1}{2}$ and $E\{\theta\}=\frac{\bar{\theta}+\underline{\theta}}{2}$. Thus, $E\left\{\frac{1-F(\theta)}{f(\theta)}\right\}=E\{\theta\}-\underline{\theta}=\frac{\bar{\theta}-\underline{\theta}}{2}$. Finally, the single peak yields $f(E\{\theta\})>\frac{1}{\bar{\theta}-\underline{\theta}}$ which implies $\frac{1-F(E\{\theta\})}{f(E\{\theta\})}=\frac{1}{2 f(E\{\theta\})}<\frac{\bar{\theta}-\underline{\theta}}{2}=E\left\{\frac{1-F(\theta)}{f(\theta)}\right\}$.
} 
the quantity assignment of an ignorant dealer. It follows that we observe downward sided bunching.

Next, suppose that the inverse hazard function is strictly Jensen convex. Consider a dealer whose liquidity is such that $\lambda(K)=p$. Condition (25) shows that $q^{0}<q=\bar{q}$ since the first order derivative of the manufacturer's objective function is negative at $q=q=\bar{q}$. Hence, there will be downward sided bunching. This situation arises because the upward movement of $q^{u}(E\{\theta\})$ in $K$ is slower than the corresponding downward movement of $q$. Hence, there exist some $K$ where the left- and upward sided limits of the preliminary schedule $q^{u}$ are in no contradiction to the monotonicity constraint $(q<\bar{q})$, but the 'slow' upward movement of $q^{u}(E\{\theta\})$ still makes bunching necessary $\left(q^{u}(E\{\theta\})<\underline{q}\right)$. If liquidity increases, there exists some interval $\left(K_{1}, K_{2}\right)$ such that the optimal quantity for the ignorant type just falls between $q$ and $\bar{q}$. In these cases, the preliminary quantity assignment rule $q^{u}(\theta)$ does not violate the monotonicity constraint and there is no bunching. For even higher $K, q^{u}(E\{\theta\})$ will exceed $\bar{q}$ and there will be upward sided bunching. This is summarized as follows.

Theorem 2 Let the inverse hazard rate be strictly Jensen convex. For any given value of p, there exist $K_{1}$ and $K_{2}$ with $K_{1}<K_{2}$ such that there is downward sided bunching for all $K<K_{1}$, there is no bunching for all $K \in\left[K_{1}, K_{2}\right]$, and there is upward sided bunching for all $K>K_{2}$.

For strictly Jensen concave inverse hazard rates, the relative movements of $q^{u}(E\{\theta\}), q$, and $\bar{q}$ are just opposite. The upward movement of $q^{u}(E\{\theta\})$ in $K$ will be faster than the corresponding downward movement of $\underline{q}$. Hence, there will exist a range $(\underline{K}, \bar{K})$ where it still holds that $q>\bar{q}$, and the preliminary quantity assignment for the ignorant type falls between $\underline{q}$ and $\bar{q}$. It follows that bunching cannot be avoided. This is summarized as our final result.

Theorem 3 Let the inverse hazard rate be strictly Jensen concave. There exist $\underline{K}$ and $\bar{K}$ such that there is downward sided bunching for all $K<\underline{K}$, double-sided bunching for all $K \in[\underline{K} ; \bar{K}]$ and upward sided bunching for all $K>\bar{K}$.

\section{Conclusion}

We have investigated supplier-credit contracting where a manufacturer does not know whether a dealer with limited liquid funds has gathered timely demand information at the contracting stage. We have found that there will be bunching in almost any case. If the probability of facing an ignorant dealer is high and the latter's liquidity is low, bunching occurs if the dealer is informed but has unfavorable information. In the contrary case where the dealer's liquidity is high relative to the likelihood that he is ignorant, bunching is only an issue for some types that are better than the average. For the intermediate cases, it depends on the curvature of the inverse hazard rate whether there is bunching for some intermediate types or no bunching at all.

Our results raise follow-up questions. In our model, the dealer is either informed or ignorant about demand of the upcoming period. Alternatively, one may assume that the dealer always has demand information, but this information is noisy. While this 
amendment will have little impact on the solution if liquidity is high, we should expect bunching to become an even more serious issue if liquidity is low. In the extreme, if the variance of the noise variable is very high and the dealer's liquidity is zero, no screening will occur. Moreover, one could extend the timing of our game and allow for several order dates. Now, the order strategies of an informed and an ignorant dealer should look different such that an additional screening device exists which may help to overcome the problems arising from being ignorant jointly with the limited liquidity restriction.

From the perspective of contract enforcement, verifiability of important variables is crucial. For instance, we have assumed that the manufacturer may always observe and verify the quantity that has been sold by the dealer. This may be possible, if the manufacturer has installed a guarantee system for his products (as is, for instance, common in the car manufacturing industry). Another setting could be that the manufacturer cannot observe the quantity that has been sold by the dealer. Then, a dealer who has reported to be ignorant about demand might claim that the assigned quantity could not be sold. In the extreme case without liquid funds at the outset, the optimal supply-credit contract would exclude such a dealer and - as a consequence - any informed dealer with unfavorable information.

From an empirical perspective, our research suggests that trade credits should come along with restrictions on the quantity assignment and with non-linear pricing schedules. We expect to see a non-linear pricing scheme with discontinuities and strong dependence of the terms of the supply-credit contract on the dealer's initial financial position.

Acknowledgments We thank two anonymous referees, Dirk Simons (the editor), Christian Laux, Stefan Reichelstein, Manfred Stadler, Uwe Walz, seminar participants at Magdeburg University and Humboldt University of Berlin for helpful comments and discussions.

\section{Appendix}

Preliminary steps. Before we formally prove the results, we restate preliminary results that are used in all the subsequent maximization problems. Consider two values $\theta>\theta^{\prime}$. If the incentive constraint (6) holds, we may write

$$
\begin{gathered}
\theta q(\theta)+r(q(\theta))-t(\theta) \geq \theta q\left(\theta^{\prime}\right)+r\left(q\left(\theta^{\prime}\right)\right)-t\left(\theta^{\prime}\right) \text { or } \frac{\Pi_{2}(\theta \mid \theta)-\Pi_{2}\left(\theta^{\prime} \mid \theta^{\prime}\right)}{\theta-\theta^{\prime}} \geq q\left(\theta^{\prime}\right) \\
\theta^{\prime} q\left(\theta^{\prime}\right)+r\left(q\left(\theta^{\prime}\right)\right)-t\left(\theta^{\prime}\right) \geq \theta^{\prime} q(\theta)+r(q(\theta))-t(\theta) \text { or } \frac{\Pi_{2}(\theta \mid \theta)-\Pi_{2}\left(\theta^{\prime} \mid \theta^{\prime}\right)}{\theta-\theta^{\prime}} \leq q(\theta) .
\end{gathered}
$$

Taking the limit, it follows that truth-telling implies $\dot{\Pi}(\theta \mid \theta)=q(\theta)$. Hence, we may write

$$
\Pi_{2}(\theta \mid \theta)=\Pi_{2}(\underline{\theta} \mid \underline{\theta})+\int_{\underline{\theta}}^{\theta} q(\vartheta) \mathrm{d} \vartheta .
$$


Since $\Pi_{2}(\theta \mid \theta)>0$ for all $\theta>\underline{\theta}$, we thus may replace the participation constraint (3) by

$$
\Pi_{2}(\underline{\theta} \mid \underline{\theta}) \geq 0
$$

Moreover, as shown by Guesnerie and Laffont (1984) or in Theorem 7.3 in Fudenberg and Tirole (1991), if $q(\theta)$ is non-decreasing in $\theta$, the incentive constraint (6) is satisfied.

Proof of Lemma 1 If $p=1$, the constraints (4), (7), and (8) are not binding. It follows that the modified participation constraint (28) must be binding in the manufacturer's optimum because otherwise the manufacturer could raise $t(m)$ for each $m$ by a positive amount and increase her profit.

Using these results, rewrite the manufacturer's objective function (2) as

$$
\int_{\underline{\theta}}^{\bar{\theta}}\left[\theta q(\theta)+r(q(\theta))-C(q(\theta))-\int_{\underline{\theta}}^{\theta} q(\vartheta) \mathrm{d} \vartheta\right] f(\theta) \mathrm{d} \theta .
$$

Integration by parts leads to the manufacturer's objective function (9) in Lemma 1. Pointwise maximization leads to the implicit rule for $q^{p=1}(\theta)$ as stated in condition (10). Finally, notice that Assumption 1 implies that $q^{p=1}(\theta)$ is nondecreasing in $\theta$.

Proof of Proposition 1 We proceed along the following steps. In Lemma 4, we show that at least one of the constraints (14) and (15) is binding in the manufacturer's optimum. We also show that in the case of positive but sufficiently low liquidity (i.e., $0<K<K_{\mathrm{LS}}$ ), the fact that the participation constraint (14) is binding (i.e. $\lambda>0$ ) implies that also the limited liquidity constraint (15) is binding (i.e., $\mu>0$ ). The final step is to show that the reverse implication also holds (Lemma 6).

Lemma 4 In the manufacturer's optimum, at least one of the constraints (14) and (15) is binding. Moreover, if $K<K_{\mathrm{LS}}$, the fact that constraint (14) is binding implies that constraint (15) is binding.

Proof of Lemma 4 Use (27) to rewrite the limited liability constraint (15) as

$$
\underline{\theta} q^{0}+r\left(q^{0}\right)-t^{0}+K=\Pi(\underline{\theta} \mid \underline{\theta})+\int_{\underline{\theta}}^{\theta} q(\vartheta) \mathrm{d} \vartheta-[E\{\theta\}-\underline{\theta}] q^{0}+K \geq 0
$$

As any increase in $\Pi(\underline{\theta} \mid \underline{\theta})$ is costly to the manufacturer, at least one of the constraints (14) and (15) will be binding in the manufacturer's optimum.

Now assume that $\Pi(\underline{\theta} \mid \underline{\theta})=0$, i.e., $\lambda>0$. If constraint (15) is slack, we are back to the LS case. Then it must necessarily hold that $K$ exceeds a lower bound $K_{\mathrm{LS}}$, 
given by

$$
\begin{aligned}
& K_{\mathrm{LS}}=-\Pi_{2}\left(s^{0} \mid \underline{\theta}\right)=-\underline{\theta} \cdot q^{\mathrm{LS}}(E\{\theta\})-r\left(q^{\mathrm{LS}}(\theta)\right)+t^{\mathrm{LS}}(E\{\theta\}) \\
&=[E\{\theta\}-\underline{\theta}] q^{\mathrm{LS}}(E\{\theta\})-\int_{\underline{\theta}}^{E\{\theta\}} q^{\mathrm{LS}}(\vartheta) \mathrm{d} \vartheta .
\end{aligned}
$$

Hence, assume that $K<K_{\mathrm{LS}}$. If $\Pi(\underline{\theta} \mid \underline{\theta})=0$ holds, it follows that the limited liquidity constraint (15) is binding too.

To continue the analysis we now consider the reverse direction, i.e., we assume that the limited liquidity constraint (15) is binding. We directly substitute the binding constraint into the objective function, thus omitting $\mu$ for the ease of presentation. Integrating by parts, we simplify the statement of the manufacturer's problem as follows.

Lemma 5 Suppose that $0<K<K_{\mathrm{LS}}$ and constraint (15) is binding. Let $\mathbf{1}_{\Sigma}$ be 1 if the statement $\Sigma$ is true and 0 otherwise. Then, the manufacturer's maximization problem can be restated as

$$
\begin{aligned}
& \max _{q(\theta), q^{0}} p \int_{\underline{\theta}}^{\bar{\theta}}\left[\theta q(\theta)+r(q(\theta))-C(q(\theta))-q(\theta)\left(\frac{1-F(\theta)}{f(\theta)}-\frac{(p-\lambda) \mathbf{1}_{\theta \leq E\{\theta\}}}{p f(\theta)}\right)\right] \\
& \quad \times f(\theta) \mathrm{d} \theta+(1-p)\left[\underline{\theta} q^{0}+r\left(q^{0}\right)-C\left(q^{0}\right)\right]-(p-\lambda)[E\{\theta\}-\underline{\theta}] q^{0}+(1-\lambda) K,
\end{aligned}
$$

subject to $q^{0}=q(E\{\theta\})$ and $q(\theta)$ non-decreasing in $\theta$.

Proof of Lemma 5 The manufacturer's Lagrangian is given as

$$
p \int_{\underline{\theta}}^{\bar{\theta}}[t(\theta)-C(q(\theta))] f(\theta) \mathrm{d} \theta+(1-p)[t(E\{\theta\})-C(q(E\{\theta\}))]+\lambda \Pi_{2}(\underline{\theta} \mid \underline{\theta})
$$

where $t(\theta)=\theta q(\theta)+r(q(\theta))-\Pi(\underline{\theta} \mid \underline{\theta})-\int_{\theta}^{\theta} q(\vartheta) \mathrm{d} \vartheta$. We use the fact that constraint (15) is binding by eliminating $\Pi_{2}(\underline{\theta} \mid \underline{\theta})$ as follows

$$
\begin{aligned}
\Pi_{2}(\underline{\theta} \mid \underline{\theta}) & =\Pi_{2}(E\{\theta\} \mid E\{\theta\})-\int_{\underline{\theta}}^{E\{\theta\}} q(\vartheta) \mathrm{d} \vartheta-t(E\{\theta\}) \\
& =[E\{\theta\}-\underline{\theta}] q^{0}-\int_{\underline{\theta}}^{E\{\theta\}} q(\vartheta) \mathrm{d} \vartheta-K .
\end{aligned}
$$

Substituting into the Lagrangian proves the Lemma. 
The following Lemma states that the Lagrange multiplier $\lambda$ is strictly positive, if $K>0$ and the limited liquidity constraint (15) is binding. Since we have previously shown that for $0<K<K_{\mathrm{LS}}$ a binding participation constraint (14) implies a binding limited liquidity constraint (15), we are now able to state that these two properties are equivalent.

Lemma 6 Suppose that $0<K<K_{\mathrm{LS}}$ and constraint (15) is binding. The optimal bunching quantity assignment $q^{0}$ is given as the solution to

$$
\begin{aligned}
& (1-p) \underline{\theta}+\left[1-p+p\left(F\left(\theta_{2}\right)-F\left(\theta_{1}\right)\right)\right]\left[r^{\prime}\left(q^{0}\right)-C^{\prime}\left(q^{0}\right)\right] \\
& \quad-p \cdot\left[\theta_{2}\left(1-F\left(\theta_{2}\right)\right)-\theta_{1}\left(1-F\left(\theta_{1}\right)\right)\right]-(p-\lambda)\left(\theta_{1}-\underline{\theta}\right)=0 .
\end{aligned}
$$

Moreover, for $0<K<K_{\mathrm{LS}}$ both Lagrange multipliers are between zero and one with $\mu=1-\lambda$. For $K=0$ we obtain $\lambda=0$ and for $K=K_{\mathrm{LS}}$ we have $\mu=0$.

Proof of Lemma 6 Ignore the monotonicity constraint and take the pointwise derivative of the objective function (29). Denote the solution to the relaxed problem by $q^{u}(\theta)$. It is determined as follows.

$$
\begin{array}{ll}
\text { If } \theta>E\{\theta\} & \theta+r^{\prime}\left(q^{u}(\theta)\right)-C^{\prime}\left(q^{u}(\theta)\right)-\frac{1-F(\theta)}{f(\theta)}=0 \\
\text { if } \theta<E\{\theta\} & \theta+r^{\prime}\left(q^{u}(\theta)\right)-C^{\prime}\left(q^{u}(\theta)\right)-\left(\frac{1-F(\theta)}{f(\theta)}-\frac{p-\lambda}{p} \frac{1}{f(\theta)}\right)=0 \\
\text { if } \theta=E\{\theta\} & (1-p)\left[\underline{\theta}+r^{\prime}\left(q^{u}(E\{\theta\})\right)-C^{\prime}\left(q^{u}(E\{\theta\})\right)\right]-(p-\lambda)[E\{\theta\}-\underline{\theta}]=0 .
\end{array}
$$

We now prove that condition (30) determines the optimal pooling quantity. Monotonicity requires $\underline{q} \leq q^{u}(E\{\theta\}) \leq \bar{q}$. If this condition is violated under the preliminary first-order conditions, we have to find an optimal bunching quantity $q^{0}$. We use a two-step procedure.

Step 1: Let $q^{0}$ be given. The maximum value function $W^{*}\left(q^{0}\right)$ is given by

$$
\begin{aligned}
W^{*}\left(q^{0}\right)= & \int_{\underline{\theta}}^{\theta_{1}}\left[\theta q^{u}(\theta)+r\left(q^{u}(\theta)\right)-C\left(q^{u}(\theta)\right)-q^{u}(\theta)\right. \\
& \left.\left(\frac{1-F(\theta)}{f(\theta)}-\frac{p-\lambda}{p} \frac{1}{f(\theta)}\right)\right] f(\theta) \mathrm{d} \theta \\
& +\int_{\theta_{1}}^{E\{\theta\}}\left[\theta q^{0}+r\left(q^{0}\right)-C\left(q^{0}\right)-q^{0}\left(\frac{1-F(\theta)}{f(\theta)}-\frac{p-\lambda}{p} \frac{1}{f(\theta)}\right)\right] f(\theta) \mathrm{d} \theta
\end{aligned}
$$




$$
\begin{aligned}
& +\int_{E\{\theta\}}^{\theta_{2}}\left[\theta q^{0}+r\left(q^{0}\right)-C\left(q^{0}\right)-q^{0} \frac{1-F(\theta)}{f(\theta)}\right] f(\theta) \mathrm{d} \theta \\
& +\int_{\theta_{2}}^{\bar{\theta}}\left[\theta q^{u}(\theta)+r\left(q^{u}(\theta)\right)-C\left(q^{u}(\theta)\right)-q^{u}(\theta) \frac{1-F(\theta)}{f(\theta)}\right] f(\theta) \mathrm{d} \theta .
\end{aligned}
$$

Step 2: Now, solve for the optimal $q^{0}$. To this end, maximize

$$
\begin{aligned}
P \equiv & p \cdot W^{*}\left(q^{0}\right)-(p-\lambda) \cdot[E\{\theta\}-\underline{\theta}] \cdot q^{0} \\
& +(1-p) \cdot\left[\underline{\theta} q^{0}+r\left(q^{0}\right)-C\left(q^{0}\right)\right]+(1-\lambda) \cdot K
\end{aligned}
$$

with respect to $q^{0}$. As a necessary maximization condition we obtain

$$
p \cdot W^{* \prime}\left(q^{0}\right)-(p-\lambda) \cdot[E\{\theta\}-\underline{\theta}]+(1-p) \cdot\left[\underline{\theta}+r^{\prime}\left(q^{0}\right)-C^{\prime}\left(q^{0}\right)\right]=0
$$

where

$$
\begin{aligned}
W^{* \prime}\left(q^{0}\right)= & \frac{p-\lambda}{p} \cdot\left[E\{\theta\}-\theta_{1}\right]-\theta_{2}\left(1-F\left(\theta_{2}\right)\right)+\theta_{1}\left(1-F\left(\theta_{1}\right)\right) \\
& +\left[r^{\prime}\left(q^{0}\right)-C^{\prime}\left(q^{0}\right)\right]\left[F\left(\theta_{2}\right)-F\left(\theta_{1}\right)\right] .
\end{aligned}
$$

Substitute (34) into (33) and rearrange to obtain

$$
\begin{aligned}
& (1-p) \underline{\theta}+\left[1-p+p\left(F\left(\theta_{2}\right)-F\left(\theta_{1}\right)\right)\right]\left[r^{\prime}\left(q^{0}\right)-C^{\prime}\left(q^{0}\right) \cdot\right] \\
& \quad-p \cdot\left[\theta_{2}\left(1-F\left(\theta_{2}\right)\right)-\theta_{1}\left(1-F\left(\theta_{1}\right)\right)\right]-(p-\lambda)\left(\theta_{1}-\underline{\theta}\right)=0 .
\end{aligned}
$$

Now, we prove the comparative statics about $\lambda$. To this end, apply the implicit-function theorem to

$$
\begin{aligned}
G^{1}\left(\lambda ; q^{0}\right)= & (1-p) \underline{\theta}+\left[r^{\prime}\left(q^{0}\right)-C^{\prime}\left(q^{0}\right)\right] \cdot\left[1-p+p\left(F\left(\theta_{2}\right)-F\left(\theta_{1}\right)\right)\right] \\
& -p \cdot\left[\theta_{2}\left(1-F\left(\theta_{2}\right)\right)-\theta_{1}\left(1-F\left(\theta_{1}\right)\right)\right]-(p-\lambda)\left(\theta_{1}-\underline{\theta}\right)=0 \\
G^{2}\left(\lambda ; q^{0}\right)= & {[E(\theta)-\underline{\theta}] \cdot q^{0}-K-\int_{\underline{\theta}}^{E(\theta)} q(\tilde{\theta}) d \tilde{\theta}=0 }
\end{aligned}
$$


to get

$$
\frac{d \lambda}{d K}=-\frac{\left|\begin{array}{cc}
G_{K}^{1} & G_{q^{0}}^{1} \\
G_{K}^{2} & G_{q^{0}}^{2}
\end{array}\right|}{\left|\begin{array}{ll}
G_{\lambda}^{1} & G_{q^{0}}^{1} \\
G_{\lambda}^{2} & G_{q^{0}}^{2}
\end{array}\right|}=\frac{-\frac{\partial^{2} P}{\partial\left(q^{0}\right)^{2}}}{\left(\theta_{1}-\underline{\theta}\right)(E\{\theta\}-\underline{\theta})}>0
$$

(where $P$ is defined in (32)), and

$$
\frac{\mathrm{d} q^{0}}{\mathrm{~d} K}=\frac{1}{(E\{\theta\}-\underline{\theta})}>0 .
$$

Compare $q$ and $\bar{q}$ as given by the respective limits of (17) and (18). To see that $\lambda(0)=0$ observe that for $K=0$ it must hold that $\theta q^{0}+r\left(q^{0}\right)-t^{0} \geq 0$ which, using the incentive constraint for an informed dealer, implies that $\underline{\theta} q(\underline{\theta})+r(q(\underline{\theta}))-t(\underline{\theta}) \geq 0$.

To see that $\mu=1-\lambda$ rewrite problem (13)-(16) using $\Pi(\underline{\theta} \mid \underline{\theta})=0$ while adding the liability constraint with its Lagrangian multiplier, $\mu$. The usual manipulations lead to the objective function

$$
\begin{array}{rl}
\max _{q(\theta), q^{0}} & p \int_{\underline{\theta}}^{\bar{\theta}}\left[\theta q(\theta)+r(q(\theta))-C(q(\theta))-q(\theta) \frac{1-F(\theta)}{f(\theta)}\right. \\
& \left.+\frac{(p-1+\mu) \mathbf{1}_{\theta \leq E\{\theta\}}}{p f(\theta)} q(\theta)\right] f(\theta) \mathrm{d} \theta \\
& +(1-p)\left[E\{\theta\} q^{0}+r\left(q^{0}\right)-C\left(q^{0}\right)\right]-\mu[E\{\theta\}-\underline{\theta}] q^{0}+\mu K .
\end{array}
$$

Comparing the problem formulation to that in Lemma 5, we immediately see that $\mu=1-\lambda$.

Proofs of Theorems 1 to 3

Definition 3 To shorten notation we define

$$
\alpha \equiv \frac{\frac{1-F(E\{\theta\})}{f(E\{\theta\}}}{E\left\{\frac{1-F(\theta)}{f(\theta)}\right\}} .
$$

Using this definition, we have $\alpha=1$ if and only if the hazard rate is Jensen linear, $\alpha<1$ if and only if the hazard rate is Jensen convex, and $\alpha>1$ if and only if the hazard rate is Jensen concave. 


\section{Lemma 7 Define}

$$
\begin{aligned}
& \hat{\lambda}=1-(1-p) \alpha \quad \text { and } \\
& \check{\lambda}=\frac{1-F(E\{\theta\})+(1-p) \alpha F(E\{\theta\})}{1-F(E\{\theta\})+\frac{1-p}{p} \alpha} .
\end{aligned}
$$

We get $q^{u}(E\{\theta\})<\bar{q}(=\bar{q},>\bar{q})$ if and only if $\lambda<\hat{\lambda}(\lambda=\hat{\lambda}, \lambda>\hat{\lambda})$ and $q^{u}(E\{\theta\})<$ $\underline{q}(=\underline{q},>\underline{q})$ if and only if $\lambda<\check{\lambda}(\lambda=\check{\lambda}, \bar{\lambda}>\check{\lambda})$.

Proof of Lemma 7 First, evaluate (19) at $q^{0}=\bar{q}$.

$$
\begin{aligned}
& (1-p)\left[\underline{\theta}+r^{\prime}(\bar{q})-C^{\prime}(\bar{q})\right]-(p-\lambda)[E\{\theta\}-\underline{\theta}] \\
& =(1-p)\left[\frac{1-F(E\{\theta\})}{f(E\{\theta\})}-E\left\{\frac{1-F(\theta)}{f(\theta)}\right\}\right]-(p-\lambda) E\left\{\frac{1-F(\theta)}{f(\theta)}\right\} \\
& =(1-p) \frac{1-F(E\{\theta\})}{f(E\{\theta\})}-(1-\lambda) E\left\{\frac{1-F(\theta)}{f(\theta)}\right\}
\end{aligned}
$$

where this expression is negative (positive, equal to zero) if and only if $\lambda<\hat{\lambda}$ ( $\lambda>$ $\hat{\lambda}, \lambda=\hat{\lambda}$.

Now, evaluate (19) at $q^{0}=\underline{q}$.

$$
\begin{aligned}
& (1-p)\left[\underline{\theta}+r^{\prime}(\bar{q})-C^{\prime}(\bar{q}]-(p-\lambda)[E\{\theta\}-\underline{\theta}]\right. \\
& \quad=(1-p) \frac{1-F(E\{\theta\})}{f(E\{\theta\})}-\frac{(1-p)(p-\lambda)}{p f(E\{\theta\})}-(1-\lambda) E\left\{\frac{1-F(\theta)}{f(\theta)}\right\}
\end{aligned}
$$

where this expression is negative (positive, equal to zero) if and only if $\lambda<\check{\lambda}$ ( $\lambda>$ $\check{\lambda}, \lambda=\check{\lambda}$ ).

Lemma 8 Comparing the two threshold values $\hat{\lambda}$ and $\check{\lambda}$ and $p$, we get

$$
\begin{array}{ll}
\alpha<1 & p<\check{\lambda}<\hat{\lambda} \\
\alpha=1 & p=\check{\lambda}=\hat{\lambda} \\
\alpha>1 & p>\check{\lambda}>\hat{\lambda}
\end{array}
$$

Proof of Lemma 8 By direct comparison of (41) and (42).

Lemma 9 Suppose $q^{u}(E\{\theta\})<\bar{q}<\underline{q}$ holds. Then, $q^{0}<\bar{q}$ if $\lambda \leq p$ and $\alpha \leq 1$. 
Proof of Lemma 9 Evaluate the first-order condition (22) at $\bar{q}$ which implies $\theta_{2}=$ $E\{\theta\}$ and $\theta_{1} \in[\underline{\theta} ; E\{\theta\}]$, such that

$$
\begin{aligned}
(1-p)\left[\underline{\theta}+r^{\prime}(\bar{q})-C^{\prime}(\bar{q})\right]+p\left[r^{\prime}(\bar{q})-C^{\prime}(\bar{q})\right]\left(F(E\{\theta\})-F\left(\theta_{1}\right)\right) \\
\quad-p E(1-F(E\{\theta\}))+p \theta_{1}\left(1-F\left(\theta_{1}\right)\right)-(p-\lambda)\left[\theta_{1}-\underline{\theta}\right] \\
=(1-p)\left(\frac{1-F(E\{\theta\})}{f(E\{\theta\})}-E\left\{\frac{1-F(\theta)}{f(\theta)}\right\}\right)-(p-\lambda)\left[\theta_{1}-\underline{\theta}\right] \\
\quad+p F(E\{\theta\})\left(E\{\theta\}+r^{\prime}(\bar{q})-C^{\prime}(\bar{q})\right]-p E\{\theta\} \\
\quad-p F\left(\theta_{1}\right)\left(\theta_{1}+r^{\prime}(\bar{q})-C^{\prime}(\bar{q})\right]+p \theta_{1} .
\end{aligned}
$$

Since

$$
\left.\frac{\partial\left[p F(\theta)\left(\theta+r^{\prime}(q)-C^{\prime}(q)\right)-p \theta\right]}{\partial \theta}=p f(\theta)\left[\theta+r^{\prime}(q)-C^{\prime}(q)\right)-\frac{1-F(\theta)}{f(\theta)}\right]=0
$$

if $q=q^{u}(\theta)$ for all $\theta \in[\underline{\theta} ; E\{\theta\}]$, and the respective second order derivative is positive, we have

$p F(E\{\theta\})\left(E\{\theta\}+r^{\prime}(\bar{q})-C^{\prime}(\bar{q})\right]-p E\{\theta\}<p F\left(\theta_{1}\right)\left(\theta_{1}+r^{\prime}(\bar{q})-C^{\prime}(\bar{q})\right]-p \theta_{1}$

such that (43) is negative if $\lambda \leq p$ and $\alpha \leq 1$.

Lemma 10 Suppose $q^{u}(E\{\theta\})>\bar{q}>\underline{q}$ holds. We get $q^{0}<\bar{q}$ if $\lambda<\hat{\lambda}$ while we get $q^{0}>\bar{q}$ if $\lambda>\hat{\lambda}$.

Proof of Lemma 10 Evaluate the first-order condition (22) at $q^{0}=\bar{q}$ which implies $\theta_{1}=\theta_{2}=E\{\theta\}$ and therefore

$$
\begin{aligned}
& (1-p)\left(\underline{\theta}+r^{\prime}(\bar{q})-C^{\prime}(\bar{q})\right)-(p-\lambda)[E\{\theta\}-\underline{\theta}] \\
& \quad=(1-p) \frac{1-F(E\{\theta\})}{f(E\{\theta\})}-(1-\lambda) E\left\{\frac{1-F(\theta)}{f(\theta)}\right\} .
\end{aligned}
$$

Expression (44) is positive for all $\lambda>\hat{\lambda}$ such that $q^{0}>\bar{q}$, negative for all $\lambda<\hat{\lambda}$ such that $q^{0}<\bar{q}$, and equal to zero if $\lambda=\hat{\lambda}$ which implies $q^{0}=\bar{q}$.

Corollary 1 Suppose $q^{u}(E\{\theta\})<\underline{q} \leq \bar{q}$ holds. We get $q^{0}<\underline{q}$ if $\lambda<\hat{\lambda}$.

Proof of Corollary 1 Evaluate the first-order condition (22) at $\underline{q}$ which implies $\theta_{2}=$ $E\{\theta\}$ and $\theta_{1} \in[\underline{\theta} ; E\{\theta\}]$. Parallel reasoning as in Lemma 10 reveals that this expression is negative if $\lambda<\hat{\lambda}$.

Proof of Theorem 1 As $\alpha=1$, we get $\hat{\lambda}=p=\check{\lambda}$. Depending on $\lambda$, three possible subcases can emerge.

- Case a: Let $\lambda$ be small such that $\lambda<\hat{\lambda}=p=\check{\lambda}$. This inequality immediately reveals that $q^{u}(E\{\theta\})<\bar{q}<q$. Lemma 9 shows that $q^{0}<\bar{q}$. Thus, there is downward sided bunching. 
- Case b: Suppose $\lambda=\hat{\lambda}=p=\check{\lambda}$, which implies $\underline{q}=\bar{q}=q^{u}(E\{\theta\})$. The preliminary first-order conditions do not violate the monotonicity requirement. They define the solution, and there is no bunching.

- Case c: Let $\lambda$ be large such that $\hat{\lambda}=p=\check{\lambda}<\lambda$, which means $\underline{q}<\bar{q}<q^{u}(E\{\theta\})$. Lemma 10 shows that $q^{0}>\bar{q}$ as $\lambda>\hat{\lambda}$, such that there is upward sided bunching.

Proof of Theorem 2 As $\alpha<1$, we get $p<\check{\lambda}<\hat{\lambda}$. Depending on $\lambda$, four possible subcases can emerge.

- Case a: Let $\lambda$ be small such that $\lambda \leq p<\check{\lambda}<\hat{\lambda}$ which implies $q^{u}(E\{\theta\})<\bar{q} \leq \underline{q}$ where the equality holds if and only if $\lambda=p$. Following Lemma 9 we get $q^{0}<\bar{q}$ or there will be downward sided bunching.

- Case b: Let $p<\lambda<\check{\lambda}<\hat{\lambda}$ which means $q^{u}(E\{\theta\})<\underline{q}<\bar{q}$. Following Corollary $1, q^{0}<q$ holds. Thus, there is downward sided bunching too.

- Case c: Let $p<\check{\lambda} \leq \lambda \leq \hat{\lambda}$ which implies $q \leq q^{u}(E\{\theta\}) \leq \bar{q}$. The monotonicity constraint is not violated and there is no bunching.

- Case d: Let $p<\check{\lambda}<\hat{\lambda}<\lambda$. Since $q<\bar{q}<q^{u}(E\{\theta\})$, Lemma 10 applies and $q^{0}>\bar{q}$ implies upward sided bunching.

Proof of Theorem 3 From $\alpha>1$ we get $\hat{\lambda}<\check{\lambda}<p$. Three different subcases have to be considered.

- Case a: Consider $\hat{\lambda}<\check{\lambda}<p<(=) \lambda$ which means $q<(=) \bar{q}<q^{u}(E\{\theta\})$. Apply Lemma 10 to get $q^{0}>\bar{q}$ or upward sided bunching.

- Case b: Consider small values of $\lambda<\hat{\lambda}<\breve{\lambda}<p$. First note that if $\alpha>1 /(1-p)$, our interval gets empty as $\hat{\lambda}=0$. Therefore suppose $\alpha<1 /(1-p)$. Condition (43) does not give clear cut evidence. At least for some neighborhood around $\alpha=1$ the same arguments as under Theorem 1 apply. If $\alpha$ increases, the sign may change such that double sided bunching occurs. Thus, we can define $\underline{K}$ and $\lambda(\underline{K})<\hat{\lambda}$ such that the first-order condition evaluated at $\bar{q}$ (condition (43)) is just equal to zero. For all $\lambda<\lambda(\underline{K})$ there is downward-sided bunching while there is double-sided bunching for $\lambda \in[\lambda(\underline{K}) ; \hat{K}]$

- Case c: Consider intermediate values of $\lambda$. There exist $\bar{K}$ with $\lambda(\bar{K}) \in[\hat{\lambda} ; p]$; $\underline{K}<\bar{K}$, such that $q^{0}<\underline{q}$ for all $K<\bar{K}$ and $q^{0}>\underline{q}$ for all $K>\bar{K}$. Thus, there is double-sided bunching for all $K \in[\underline{K} ; \bar{K}]$, there. is downward sided bunching for all $K<\underline{K}$, and there is upward sided bunching for all $K>\bar{K}$.

\section{References}

Cachon G (2003) Supply chain coordination with contracts. In: Graves S, de Kok AG (eds) Handbooks in operations research and management science: Supply chain management: design, coordination and operation. North Holland, Amsterdam, pp 229-339

Cachon G, Lariviere MA (2001) Contracting to assure supply: How to share demand forecasts in a supply chain. Manag Sci 47:629-646

Che YK, Gale I (2000) The optimal mechanism for selling to a budget-constrained buyer. J Econ Theor 92:198-233 
Dye RA (1985) Disclosure of nonproprietary information. J Account Res 23(1):123-145

Fudenberg D, Tirole J (1991) Game theory. MIT Press, Cambridge

Guesnerie R, Laffont JJ (1984) A complete solution to a class of principal-agent problems with an application to the control of a self-managed firm. J Pub Econ 25:329-369

Harris M, Kriebel C, Raviv A (1982) Asymmetric information, incentives, and intrafirm resource allocation. Manag Sci 28:604-620

Jullien B (2000) Participation constraints in adverse selection models. J Econ Theor 93:1-47

Jung WO, Kwon YK (1988) Disclosure when the market is unsure of information endowment of managers. J Account Res 26:146-153

Lewis TR, Sappington DEM (1989) Countervailing incentives in agency problems. J Econ Theor 61:294313

Lewis TR, Sappington DEM (1993) Ignorance in agency problems. J Econ Theor 61:169-183

Lewis TR, Sappington DEM (2000) Motivating wealth-constrained actors. Am Econ Rev 90:944-960

Lewis TR, Sappington DEM (2001) Optimal contracting with private knowledge of wealth and ability. Rev Econ Stud 68(1):21-44

Myerson RM (1982) Optimal coordination mechanisms in generalized principal-agent problems. J Math Econ 10(1):67-81

Penno MC (1997) Information quality and voluntary disclosure. Account Rev 72(2):275-284

Sappington DEM (1983) Limited liability between principal and agent. J Econ Theor 29:1-21 